\title{
Torus-invariant prime ideals in quantum matrices, totally nonnegative cells and symplectic leaves
}

\author{
K. R. Goodearl • S. Launois · T. H. Lenagan
}

Received: 5 October 2009 / Accepted: 2 April 2010 / Published online: 27 April 2010

(C) The Author(s) 2010. This article is published with open access at Springerlink.com

\begin{abstract}
The algebra of quantum matrices of a given size supports a rational torus action by automorphisms. It follows from work of Letzter and the first named author that to understand the prime and primitive spectra of this algebra, the first step is to understand the prime ideals that are invariant under the torus action. In this paper, we prove that a family of quantum minors is the set of all quantum minors that belong to a given torus-invariant prime ideal of a quantum matrix algebra if and only if the corresponding family of minors defines a non-empty totally nonnegative cell in the space of totally nonnegative real matrices of the appropriate size. As a corollary, we obtain explicit generating sets of quantum minors for the torus-invariant prime ideals of quantum matrices in the case where the quantisation parameter $q$ is transcendental over $\mathbb{Q}$.
\end{abstract}

Keywords Quantum matrices · Torus-invariant prime ideals · Quantum minors · Totally nonnegative cells $\cdot$ Symplectic leaves

Mathematics Subject Classification (2000) $\quad$ 16W35 $\cdot$ 15A48 $\cdot 17 \mathrm{~B} 37 \cdot 17 \mathrm{~B} 63 \cdot 20 \mathrm{G} 42 \cdot$ $53 \mathrm{D} 17$

The research of K. R. Goodearl was supported by a grant from the National Science Foundation (USA). The research of S. Launois was supported by a Marie Curie European Reintegration Grant within the 7th European Community Framework Programme.

K. R. Goodearl

Department of Mathematics, University of California, Santa Barbara, CA 93106, USA

e-mail: goodearl@math.ucsb.edu

S. Launois $(\varangle)$

School of Mathematics, Statistics and Actuarial Science, University of Kent,

Canterbury, Kent CT2 7NF, UK

e-mail: S.Launois@kent.ac.uk

T. H. Lenagan

Maxwell Institute for Mathematical Sciences, School of Mathematics, University of Edinburgh, James Clerk Maxwell Building, King's Buildings, Mayfield Road, Edinburgh EH9 3JZ, Scotland, UK e-mail: tom@maths.ed.ac.uk 


\section{Introduction}

In recent publications, the same combinatorial description has arisen for three separate objects of interest: torus-invariant prime ideals in quantum matrix algebras $\mathcal{O}_{q}\left(\mathcal{M}_{m, p}(\mathbb{K})\right)$ [6], torusorbits of symplectic leaves in matrix Poisson varieties $\mathcal{M}_{m, p}(\mathbb{C})$ [2], and totally nonnegative cells in spaces $\mathcal{M}_{m}^{\geq 0}, p(\mathbb{R})$ of totally nonnegative matrices [22]. Connections between the second and third of these objects were developed in [9]. Here we close the circle by linking the first and second objects. More detail follows.

Many quantum algebras have a natural action by an algebraic torus, and a key ingredient in the study of the structure of these algebras is an understanding of the torus-invariant objects. For example, the Stratification Theory of Letzter and the first named author [13] shows that, in the generic case, a complete understanding of the prime spectrum of the quantised coordinate ring of $m \times p$ matrices, $\mathcal{O}_{q}\left(\mathcal{M}_{m, p}(\mathbb{K})\right)$, would start by classifying the (finitely many) prime ideals invariant under the natural action of the torus $\mathcal{H}=\left(\mathbb{K}^{\times}\right)^{m+p}$. In [6], Cauchon succeeded in counting the number of $\mathcal{H}$-invariant prime ideals in $\mathcal{O}_{q}\left(\mathcal{M}_{m, p}(\mathbb{K})\right)$. His method involved a bijection between certain diagrams, now known as Cauchon diagrams, and the $\mathcal{H}$-invariant primes. Considerable progress in the understanding of quantum matrices has been made since that time by using Cauchon diagrams.

The semiclassical limit of the quantum matrix algebras $\mathcal{O}_{q}\left(\mathcal{M}_{m, p}(\mathbb{K})\right)$ is the classical coordinate ring of the matrix variety $\mathcal{M}_{m, p}(\mathbb{K})$, endowed with a Poisson bracket that encodes the nature of the quantum deformation which leads to quantum matrices. As a result, the variety $\mathcal{M}_{m, p}(\mathbb{K})$ is endowed with a Poisson structure. In the complex case $(\mathbb{K}=\mathbb{C})$, a natural action of the torus $\mathcal{H}=\left(\mathbb{C}^{\times}\right)^{m+p}$ leads to a stratification of the variety $\mathcal{M}_{m, p}(\mathbb{C})$ via $\mathcal{H}$-orbits of symplectic leaves. In [2], Brown, Yakimov and the first named author showed that there are finitely many such $\mathcal{H}$-orbits of symplectic leaves. Each $\mathcal{H}$-orbit is defined by certain rank conditions on submatrices. The classification is given in terms of certain permutations from the relevant symmetric group with restrictions arising from the Bruhat order.

The totally nonnegative part of the space $\mathcal{M}_{m, p}(\mathbb{R})$ of real $m \times p$ matrices consists of those matrices whose minors are all nonnegative. One can specify a cell decomposition of the set $\mathcal{M}_{\bar{m}}^{\geq 0},(\mathbb{R})$ of totally nonnegative matrices by specifying exactly which minors are to be zero/non-zero. In [22], Postnikov classified the non-empty cells by means of a bijection with certain diagrams, known as Le-diagrams.

The interesting observation from the point of view of this work is that in each of the above three sets of results, the combinatorial objects that arise turn out to be the same! The definitions of Cauchon diagrams and Le-diagrams are the same, and the restricted permutations arising in the Brown-Goodearl-Yakimov study can be seen to correspond to Cauchon/Le diagrams via the notion of pipe dreams.

Once one is aware of these combinatorial connections, the suggestion arises that there should be a connection between torus-invariant prime ideals, torus-orbits of symplectic leaves and totally nonnegative cells.

In [9], we study this connection, and prove that a family of minors defines a non-empty totally nonnegative cell in the space of totally nonnegative matrices if and only if this family is exactly the set of minors that vanish on the closure of a certain torus-orbit of symplectic leaves in the matrix Poisson variety. In the present note, we complete the picture and study the quantum case. Our main result is the following.

Theorem 4.2 Let $\mathcal{F}$ be a family of minors in the coordinate ring of the affine variety $\mathcal{M}_{m, p}(\mathbb{C})$, for some positive integers $m, p$, and let $\mathcal{F}_{q}$ be the corresponding family of quantum 
minors in $\mathcal{O}_{q}\left(\mathcal{M}_{m, p}(\mathbb{K})\right)$, where $\mathbb{K}$ is a field and $q \in \mathbb{K}^{\times}$is a non-root of unity. Then the following are equivalent:

1. The totally nonnegative cell associated to $\mathcal{F}$ in $\mathcal{M} \geq 0, p(\mathbb{R})$ is non-empty.

2. $\mathcal{F}$ is the set of all minors that vanish on the closure of some $\left(\mathbb{C}^{\times}\right)^{m+p}$-orbit of symplectic leaves in $\mathcal{M}_{m, p}(\mathbb{C})$.

3. $\mathcal{F}_{q}$ is the set of all quantum minors that belong to some $\left(\mathbb{K}^{\times}\right)^{m+p}$-invariant prime ideal in $\mathcal{O}_{q}\left(\mathcal{M}_{m, p}(\mathbb{K})\right)$.

(The torus actions in (2) and (3) are standard, and are recalled below.)

The proof of this result (see Sect. 4) relies on two algorithms, the deleting-derivations algorithm and its inverse the restoration algorithm, that were first developed for use in quantum matrices [5,6,17]. We note that recently and independently Casteels [3] has developed graph-theoretic methods (also based on the restoration algorithm) to compute the set of quantum minors that belong to a torus-invariant prime in $\mathcal{O}_{q}\left(\mathcal{M}_{m, p}(\mathbb{K})\right)$.

The sets of minors that vanish on the closure of a torus-orbit of symplectic leaves in $\mathcal{M}_{m, p}(\mathbb{C})$ have been explicitly described in [9], based on results of Fulton [7] and BrownGoodearl-Yakimov [2]. Further, Yakimov proved that the above sets of minors generate the ideals of polynomial functions vanishing on closures of torus-orbits of symplectic leaves [23]. As a consequence of the above theorem, we obtain explicit descriptions of the sets of quantum minors that belong to a torus-invariant prime in $\mathcal{O}_{q}\left(\mathcal{M}_{m, p}(\mathbb{K})\right)$ (see Theorem 4.4).

The importance of understanding the sets $\mathcal{F}_{q}$ rests on the conjecture of the first and third named authors that, in the generic case ( $q$ not a root of unity), all torus-invariant prime ideals in $\mathcal{O}_{q}\left(\mathcal{M}_{m, p}(\mathbb{K})\right)$ are generated by quantum minors [10]. In [16], the second named author proved this conjecture when the base field $\mathbb{K}$ is the field of complex numbers and the quantisation parameter $q$ is transcendental over the rationals. We extend that result here to arbitrary base fields of characteristic zero (Theorem 1.5). Consequently, in that case we deduce from the above results explicit generating sets of quantum minors for the torus-invariant prime ideals of $\mathcal{O}_{q}\left(\mathcal{M}_{m, p}(\mathbb{K})\right)$. A different approach to this result, applicable to many quantized coordinate algebras, has been recently and independently developed by Yakimov in [23]. Explicit generating sets for torus-invariant prime ideals in general will, of course, also follow if and when the above conjecture is established.

Throughout this paper, we use the following conventions:

- $\mathbb{N}$ denotes the set of natural numbers, and we set $\mathbb{C}^{\times}:=\mathbb{C} \backslash\{0\}$.

- If $I$ is any non-empty finite subset of $\mathbb{N}$, then $|I|$ denotes its cardinality.

- $\mathbb{K}$ is a field, $\mathbb{K}^{\times}:=\mathbb{K} \backslash\{0\}$ and $q \in \mathbb{K}^{\times}$is not a root of unity.

- $m$ and $p$ are two positive integers.

- If $k$ is a positive integer, then $S_{k}$ denotes the group of permutations of $\llbracket 1, k \rrbracket:=\{1, \ldots, k\}$.

- Let $K$ be a $\mathbb{K}$-algebra and $M=\left(x_{i, \alpha}\right) \in \mathcal{M}_{m, p}(K)$. If $I \subseteq \llbracket 1, m \rrbracket$ and $\Lambda \subseteq \llbracket 1, p \rrbracket$ with $|I|=|\Lambda|=k \geq 1$, then we denote by $[I \mid \Lambda]_{q}(M)$ the corresponding quantum minor of $M$. This is the element of $K$ defined by:

$$
[I \mid \Lambda]_{q}(M)=\left[i_{1}, \ldots, i_{k} \mid \alpha_{1}, \ldots, \alpha_{k}\right]_{q}:=\sum_{\sigma \in S_{k}}(-q)^{l(\sigma)} x_{i_{1}, \alpha_{\sigma(1)}} \cdots x_{i_{k}, \alpha_{\sigma(k)}},
$$

where $I=\left\{i_{1}, \ldots, i_{k}\right\}, \Lambda=\left\{\alpha_{1}, \ldots, \alpha_{k}\right\}$ and $l(\sigma)$ denotes the length of the $k$-permutation $\sigma$. Also, it is convenient to allow the empty minor: $[\emptyset \mid \emptyset]_{q}(M):=1 \in K$. Whenever we write a quantum minor in the form $\left[i_{1}, \ldots, i_{k} \mid \alpha_{1}, \ldots, \alpha_{k}\right]_{q}$, we tacitly assume that the row and column indices are listed in ascending order, that is, $i_{1}<\cdots<i_{k}$ and $\alpha_{1}<\cdots<\alpha_{k}$. 


\section{$1 \mathcal{H}$-prime ideals of $\mathcal{O}_{q}\left(\mathcal{M}_{m, p}(\mathbb{K})\right)$}

\subsection{Quantum matrices}

We denote by $R:=\mathcal{O}_{q}\left(\mathcal{M}_{m, p}(\mathbb{K})\right)$ the standard quantisation of the ring of regular functions on $m \times p$ matrices with entries in $\mathbb{K}$; the algebra $R$ is the $\mathbb{K}$-algebra generated by the $m \times p$ indeterminates $X_{i, \alpha}$, for $1 \leq i \leq m$ and $1 \leq \alpha \leq p$, subject to the following relations:

$$
\begin{array}{lll}
X_{i, \beta} X_{i, \alpha}=q^{-1} X_{i, \alpha} X_{i, \beta}, & & (\alpha<\beta) ; \\
X_{j, \alpha} X_{i, \alpha}=q^{-1} X_{i, \alpha} X_{j, \alpha}, & & (i<j) ; \\
X_{j, \beta} X_{i, \alpha}=X_{i, \alpha} X_{j, \beta}, & & (i<j, \alpha>\beta) ; \\
X_{j, \beta} X_{i, \alpha}=X_{i, \alpha} X_{j, \beta}-\left(q-q^{-1}\right) X_{i, \beta} X_{j, \alpha}, & & (i<j, \alpha<\beta) .
\end{array}
$$

It is well known that $R$ can be presented as an iterated Ore extension over $\mathbb{K}$, with the generators $X_{i, \alpha}$ adjoined in lexicographic order. Thus, the ring $R$ is a noetherian domain; its skew-field of fractions is denoted by $F$ or Fract $R$. Moreover, since $q$ is not a root of unity, it follows from [12, Theorem 3.2] that all prime ideals of $R$ are completely prime.

The quantum minors in $R$ are the quantum minors of the matrix $\left(X_{i, \alpha}\right) \in \mathcal{M}_{m, p}(R)$. To simplify the notation, we denote by $[I \mid \Lambda]_{q}$ the quantum minor of $R$ associated to the row-index set $I$ and the column-index set $\Lambda$.

It is easy to check that the torus $\mathcal{H}:=\left(\mathbb{K}^{\times}\right)^{m+p}$ acts on $R$ by $\mathbb{K}$-algebra automorphisms via:

$$
\left(a_{1}, \ldots, a_{m}, b_{1}, \ldots, b_{p}\right) . X_{i, \alpha}=a_{i} b_{\alpha} X_{i, \alpha} \quad \text { for all } \quad(i, \alpha) \in \llbracket 1, m \rrbracket \times \llbracket 1, p \rrbracket .
$$

We refer to this action as the standard action of $\left(\mathbb{K}^{\times}\right)^{m+p}$ on $\mathcal{O}_{q}\left(\mathcal{M}_{m, p}(\mathbb{K})\right)$. Recall that an $\mathcal{H}$-prime ideal of $R$ is a proper $\mathcal{H}$-invariant ideal $P$ such that whenever $P$ contains a product $I J$ of two $\mathcal{H}$-invariant ideals, it must contain either $I$ or $J$. As $q$ is not a root of unity, it follows from $[13,5.7]$ that there are only finitely many $\mathcal{H}$-primes in $R$ and that every $\mathcal{H}$-prime is completely prime. Hence, the $\mathcal{H}$-prime ideals of $R$ coincide with the $\mathcal{H}$-invariant primes. We denote by $\mathcal{H}-\operatorname{Spec}(R)$ the set of $\mathcal{H}$-primes of $R$.

\section{$1.2 \mathcal{H}$-primes and Cauchon diagrams}

In [6], Cauchon showed that his theory of deleting-derivations can be applied to the iterated Ore extension $R$. As a consequence, he was able to parametrise the set $\mathcal{H}$-Spec $(R)$ in terms of combinatorial objects called Cauchon diagrams.

Definition 1.1 [6] An $m \times p$ Cauchon diagram $C$ is simply an $m \times p$ grid consisting of $m p$ squares in which certain squares are coloured black. We require that the collection of black squares have the following property: If a square is black, then either every square strictly to its left is black or every square strictly above it is black (For an example, see Fig. 1).

We denote by $\mathcal{C}_{m, p}$ the set of $m \times p$ Cauchon diagrams.

Note that we will often identify an $m \times p$ Cauchon diagram with the set of coordinates of its black boxes. Indeed, if $C \in \mathcal{C}_{m, p}$ and $(i, \alpha) \in \llbracket 1, m \rrbracket \times \llbracket 1, p \rrbracket$, we will say that $(i, \alpha) \in C$ if the box in row $i$ and column $\alpha$ of $C$ is black.

Recall [6, Corollaire 3.2.1] that Cauchon has constructed (using the deleting-derivations algorithm) a bijection between $\mathcal{H}-\operatorname{Spec}\left(\mathcal{O}_{q}\left(\mathcal{M}_{m, p}(\mathbb{K})\right)\right)$ and the collection $\mathcal{C}_{m, p}$. We discuss this bijection in more detail in Sect. 4.

Notation 1.2 Let $C \in \mathcal{C}_{m, p}$. We denote by $J_{C}$ the unique $\mathcal{H}$-prime ideal of $R$ corresponding to the Cauchon diagram $C$ under the above bijection. 


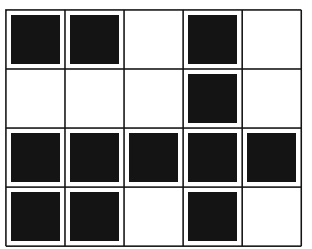

Fig. 1 An example of a $4 \times 5$ Cauchon diagram

At this point, it is worth recalling that Cauchon diagrams are also closely related to restricted permutations. More precisely, set

$$
\mathcal{S}=S_{m+p}^{[-p, m]}:=\left\{w \in S_{m+p} \mid-p \leq w(i)-i \leq m \text { for all } i=1,2, \ldots, m+p\right\} .
$$

The set $\mathcal{S}$ is an initial interval of the symmetric group $S_{m+p}$ endowed with the Bruhat order. Namely, we have [18, Proposition 1.3], [2, Lemma 3.12]:

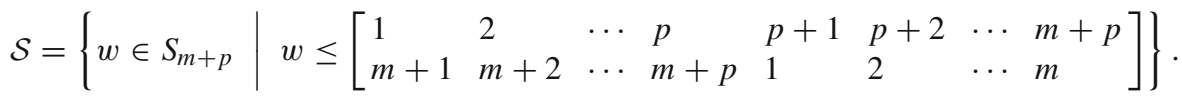

It was proved in [18, Corollary 1.5] that the cardinality of $\mathcal{S}$ is equal to the number of $m \times p$ Cauchon diagrams. Note that one can construct an explicit bijection between these two sets by using the concept of pipe-dreams. (See [22, Sect. 19].)

\subsection{Generators of $\mathcal{H}$-prime ideals of $\mathcal{O}_{q}\left(\mathcal{M}_{m, p}(\mathbb{K})\right)$}

In [10], the first and third named authors conjectured that all $\mathcal{H}$-primes in $R$ are generated by quantum minors. (Of course, any prime generated by quantum minors is an $\mathcal{H}$-prime, since every quantum minor of $R$ is an $\mathcal{H}$-eigenvector.) They proved this conjecture in the case where $m, p \leq 3[10,11]$. In [16, Théorème 3.7.2], the second named author proved this conjecture in the case where $\mathbb{K}=\mathbb{C}$ and the quantisation parameter $q$ is transcendental over $\mathbb{Q}$. The result can then be extended to arbitrary base fields of characteristic 0 (keeping $q$ transcendental over $\mathbb{Q}$ ), as we show below.

Note that the above conjecture is still open when we only assume that $q \in \mathbb{K}^{\times}$is not a root of unity.

Although in [17] an algorithm was developed that constructs, starting only from a Cauchon diagram $C$, all of the quantum minors that belong to the $\mathcal{H}$-prime ideal $J_{C}$, it is not easy to identify the families of quantum minors that generate $\mathcal{H}$-prime ideals. Casteels [3] has recently developed a graph theoretic method in order to compute these families.

In [2, Theorem 4.2], Brown, Yakimov and the first named author described the $\mathcal{H}$-orbits of symplectic leaves of $\mathcal{M}_{m, p}(\mathbb{C})$ in terms of the vanishing and nonvanishing of explicit sets of minors. Following the philosophy that symplectic leaves of $\mathcal{M}_{m, p}(\mathbb{C})$ should correspond bijectively to primitive ideals of $\mathcal{O}_{q}\left(\mathcal{M}_{m, p}(\mathbb{C})\right)$, they conjectured that a set of minors defines the closure of an $\mathcal{H}$-orbit of symplectic leaves if and only if the corresponding set of quantum minors generates an $\mathcal{H}$-prime ideal in $\mathcal{O}_{q}\left(\mathcal{M}_{m, p}(\mathbb{C})\right)$ (see $\left.[2,0.2]\right)$. This conjecture is proved here (see Theorem 4.4).

Lemma 1.3 Let $K_{1} \subseteq K_{2}$ be infinite fields, and let $A$ be a noetherian $K_{2}$-algebra supporting a rational action of a torus $H_{2}=\left(K_{2}^{\times}\right)^{r}$ by $K_{2}$-algebra automorphisms. Set $H_{1}:=\left(K_{1}^{\times}\right)^{r}$, which acts on $A$ by restriction of the $H_{2}$-action. Then the $H_{1}$-prime ideals of A coincide with the $\mathrm{H}_{2}$-prime ideals.

Proof By [1, Proposition II.2.9], every $H_{2}$-prime of $A$ is prime, and consequently $H_{1}$-prime. 
Rationality of the $H_{2}$-action on $A$ implies that $A$ has a $K_{2}$-basis $\left(a_{i}\right)_{i \in I}$ of $H_{2}$-eigenvectors whose $H_{2}$-eigenvalues are rational characters of $H_{2}$ [1, Theorem II.2.7]. If $\left(\kappa_{j}\right)_{j \in J}$ is a basis for $K_{2}$ over $K_{1}$, then $\left(\kappa_{j} a_{i}\right)_{i \in I, j \in J}$ is a $K_{1}$-basis for $A$, consisting of $H_{2}$-eigenvectors with rational $H_{2}$-eigenvalues. Observe that the restriction to $H_{1}$ of any rational character of $H_{2}$ is a rational character of $H_{1}$. Consequently, each $\kappa_{j} a_{i}$ is an $H_{1}$-eigenvector whose $H_{1}$-eigenvalue is a rational character of $H_{1}$. Thus, the $H_{1}$-action on $A$ is rational.

Now any $H_{1}$-prime $P$ of $A$ is prime by [1, Proposition II.2.9]. Consider the induced action of $H_{2}$ on $\operatorname{Spec}(A)$ by homeomorphisms, and let $S$ be the stabilizer of $P$ in $H_{2}$ under this action. It follows from [1, Lemma II.2.8] that $S$ is a Zariski-closed subgroup of $\mathrm{H}_{2}$. On the other hand, $S \supseteq H_{1}$, and $H_{1}$ is Zariski-dense in $H_{2}$ because $K_{1}$ is infinite. Therefore $S=H_{2}$, whence $P$ is an $H_{2}$-prime, as required.

Proposition 1.4 Let $K_{1} \subseteq K_{2}$ be fields, $q \in K_{1}^{\times}$a non-root of unity, and identify the algebra $\mathcal{O}_{q}\left(\mathcal{M}_{m, p}\left(K_{2}\right)\right)$ with $\mathcal{O}_{q}\left(\mathcal{M}_{m, p}\left(K_{1}\right)\right) \otimes_{K_{1}} K_{2}$. Set $H_{i}:=\left(K_{i}^{\times}\right)^{m+p}$ for $i=1,2$, and let $H_{i}$ act on $\mathcal{O}_{q}\left(\mathcal{M}_{m, p}\left(K_{i}\right)\right)$ by $K_{i}$-algebra automorphisms in the standard way. Then the rule $P \mapsto P \otimes_{K_{1}} K_{2}$ gives a bijection

$$
H_{1}-\operatorname{Spec} \mathcal{O}_{q}\left(\mathcal{M}_{m, p}\left(K_{1}\right)\right) \longrightarrow H_{2}-\operatorname{Spec} \mathcal{O}_{q}\left(\mathcal{M}_{m, p}\left(K_{2}\right)\right) .
$$

Proof Set $A_{i}:=\mathcal{O}_{q}\left(\mathcal{M}_{m, p}\left(K_{i}\right)\right)$, and recall that $H_{i}$ acts rationally on $A_{i}$. By [1, Theorem II.6.4], every $H_{i}$-prime $P$ of $A_{i}$ is strongly $H_{i}$-rational in the sense that $Z\left(\text { Fract } A_{i} / P\right)^{H_{i}}=$ $K_{i}$. (See the proof of [1, Theorem II.5.14] for a verification of the required hypotheses.)

The action of $H_{1}$ on $A_{1}$ extends naturally to an action of $H_{1} \equiv H_{1} \times\langle$ id $\rangle$ on $A_{2}$ (by $K_{2}$-algebra automorphisms), and it follows from [10, Proposition 3.3] that the rule $P \mapsto P \otimes_{K_{1}} K_{2}$ gives a bijection $H_{1}-\operatorname{Spec}\left(A_{1}\right) \rightarrow H_{1}-\operatorname{Spec}\left(A_{2}\right)$. In view of Lemma 1.3, $H_{1}-\operatorname{Spec}\left(A_{2}\right)=H_{2}-\operatorname{Spec}\left(A_{2}\right)$, and the proposition is proved.

Theorem 1.5 Assume that $\operatorname{char}(\mathbb{K})=0$ and that $q$ is transcendental over $\mathbb{Q}$. Then every $\mathcal{H}$-prime of $\mathcal{O}_{q}\left(\mathcal{M}_{m, p}(\mathbb{K})\right)$ is generated, as a left (or right) ideal, by the quantum minors that it contains.

Proof First, consider the subfield $K_{1}:=\mathbb{Q}(q) \subseteq \mathbb{K}$, and identify $\mathcal{O}_{q}\left(\mathcal{M}_{m, p}(\mathbb{K})\right)$ with $\mathcal{O}_{q}\left(\mathcal{M}_{m, p}\left(K_{1}\right)\right) \otimes_{K_{1}} \mathbb{K}$. Set $H_{1}:=\left(K_{1}^{\times}\right)^{m+p}$, with the standard action on $\mathcal{O}_{q}\left(\mathcal{M}_{m, p}\left(K_{1}\right)\right)$. By Proposition 1.4, any $\mathcal{H}$-prime of $\mathcal{O}_{q}\left(\mathcal{M}_{m, p}(\mathbb{K})\right)$ has the form $P \otimes_{K_{1}} \mathbb{K}$ for some $H_{1}$-prime $P$ of $\mathcal{O}_{q}\left(\mathcal{M}_{m, p}\left(K_{1}\right)\right)$. Hence, it suffices to show that $P$ is generated, as a left (or right) ideal of $\mathcal{O}_{q}\left(\mathcal{M}_{m, p}\left(K_{1}\right)\right)$, by the quantum minors it contains.

Now identify $K_{1}$ with a subfield of $\mathbb{C}$, and set $H_{2}:=\left(\mathbb{C}^{\times}\right)^{m+p}$, with the standard action on $\mathcal{O}_{q}\left(\mathcal{M}_{m, p}(\mathbb{C})\right)$. By Proposition $1.4, P \otimes_{K_{1}} \mathbb{C}$ is an $H_{2}$-prime of $\mathcal{O}_{q}\left(\mathcal{M}_{m, p}(\mathbb{C})\right)$, and thus by [16, Théorème 3.7.2], $P \otimes_{K_{1}} \mathbb{C}$ is generated, as a left (or right) ideal of $\mathcal{O}_{q}\left(\mathcal{M}_{m, p}(\mathbb{C})\right.$ ), by the set $X$ of quantum minors it contains. Note that $X$ is also the set of quantum minors contained in $P$, and let $P^{\prime}$ be the left ideal of $\mathcal{O}_{q}\left(\mathcal{M}_{m, p}\left(K_{1}\right)\right)$ generated by $X$. Then $P^{\prime} \otimes_{K_{1}} \mathbb{C}=P \otimes_{K_{1}} \mathbb{C}$, and consequently $P^{\prime}=P$. Therefore $P$ is generated as a left ideal by $X$, and similarly as a right ideal.

\section{$2 \mathcal{H}$-orbits of symplectic leaves in $\mathcal{M}_{m, p}(\mathbb{C})$}

In this section, we study the standard Poisson structure of the coordinate ring $\mathcal{O}_{q}\left(\mathcal{M}_{m, p}(\mathbb{C})\right)$ coming from the commutators of $\mathcal{O}_{q}\left(\mathcal{M}_{m, p}(\mathbb{C})\right)$. Recall that a Poisson algebra (over $\left.\mathbb{C}\right)$ is a commutative $\mathbb{C}$-algebra $A$ equipped with a Lie bracket $\{-,-\}$ which is a derivation (for the 
associative multiplication) in each variable. The derivations $\{a,-\}$ on $A$ are called Hamiltonian derivations. When $A$ is the algebra of complex-valued $C^{\infty}$ functions on a smooth affine variety $V$, one can use Hamiltonian derivations in order to define Hamiltonian paths in $V$. A Hamiltonian path in $V$ is a smooth path $\gamma:[0,1] \rightarrow V$ such that there exists $f \in C^{\infty}(V)$ with $\frac{d \gamma}{d t}(t)=\xi_{f}(\gamma(t))$ for all $0<t<1$, where $\xi_{f}$ denotes the vector field associated to the Poisson derivation $\{f,-\}$. It is easy to check that the relation "connected by a piecewise Hamiltonian path" is an equivalence relation. The equivalence classes of this relation are called the symplectic leaves of $V$; they form a partition of $V$.

A Poisson ideal of $A$ is any ideal $I$ such that $\{A, I\} \subseteq I$, and a Poisson prime ideal is any prime ideal which is also a Poisson ideal. The set of Poisson prime ideals in $A$ forms the Poisson prime spectrum, denoted $\operatorname{PSpec}(A)$, which is given the relative Zariski topology inherited from $\operatorname{Spec}(A)$.

\subsection{The Poisson algebra $\mathcal{O}\left(\mathcal{M}_{m, p}(\mathbb{C})\right)$}

Denote by $\mathcal{O}\left(\mathcal{M}_{m, p}(\mathbb{C})\right)$ the coordinate ring of the variety $\mathcal{M}_{m, p}(\mathbb{C})$; note that $\mathcal{O}\left(\mathcal{M}_{m, p}(\mathbb{C})\right)$ is a (commutative) polynomial algebra in $m p$ indeterminates $Y_{i, \alpha}$ with $1 \leq i \leq m$ and $1 \leq \alpha \leq p$.

The variety $\mathcal{M}_{m, p}(\mathbb{C})$ is a Poisson variety: there is a unique Poisson bracket on the coordinate ring $\mathcal{O}\left(\mathcal{M}_{m, p}(\mathbb{C})\right)$ determined by the following data. For all $(i, \alpha)<(k, \gamma)$, we set:

$$
\left\{Y_{i, \alpha}, Y_{k, \gamma}\right\}= \begin{cases}Y_{i, \alpha} Y_{k, \gamma} & \text { if } i=k \text { and } \alpha<\gamma \\ Y_{i, \alpha} Y_{k, \gamma} & \text { if } i<k \text { and } \alpha=\gamma \\ 0 & \text { if } i<k \text { and } \alpha>\gamma \\ 2 Y_{i, \gamma} Y_{k, \alpha} & \text { if } i<k \text { and } \alpha<\gamma\end{cases}
$$

This is the standard Poisson structure on the affine variety $\mathcal{M}_{m, p}(\mathbb{C})$ (cf. $\left.[2, \S 1.5]\right)$; the Poisson algebra structure on $\mathcal{O}\left(\mathcal{M}_{m, p}(\mathbb{C})\right)$ is the semiclassical limit of the noncommutative algebras $\mathcal{O}_{q}\left(\mathcal{M}_{m, p}(\mathbb{C})\right)$.

As with quantum minors in $\mathcal{O}_{q}\left(\mathcal{M}_{m, p}(\mathbb{K})\right)$, we abbreviate the notation for the minors of the matrix $Y=\left(Y_{i, \alpha}\right)$, writing $[I \mid \Lambda]:=[I \mid \Lambda](Y)$.

Note that the Poisson bracket on $\mathcal{O}\left(\mathcal{M}_{m, p}(\mathbb{C})\right)$ extends uniquely to a Poisson bracket on $\mathcal{C}^{\infty}\left(\mathcal{M}_{m, p}(\mathbb{C})\right)$, so that $\mathcal{M}_{m, p}(\mathbb{C})$ can be viewed as a Poisson manifold. Hence, $\mathcal{M}_{m, p}(\mathbb{C})$ can be decomposed as the disjoint union of its symplectic leaves.

\subsection{Torus action}

Notice that the torus $\mathcal{H}:=\left(\mathbb{C}^{\times}\right)^{m+p}$ acts on $\mathcal{O}\left(\mathcal{M}_{m, p}(\mathbb{C})\right)$ by Poisson automorphisms via:

$$
\left(a_{1}, \ldots, a_{m}, b_{1}, \ldots, b_{p}\right) . Y_{i, \alpha}=a_{i} b_{\alpha} Y_{i, \alpha} \text { for all }(i, \alpha) \in \llbracket 1, m \rrbracket \times \llbracket 1, p \rrbracket .
$$

We denote by $\mathcal{H}$-PSpec $\left(\mathcal{O}\left(\mathcal{M}_{m, p}(\mathbb{C})\right)\right)$ the set of those Poisson primes of $\mathcal{O}\left(\mathcal{M}_{m, p}(\mathbb{C})\right)$ that are invariant under this action of $\mathcal{H}$. Note that $\mathcal{H}$ is acting rationally on $\mathcal{O}\left(\mathcal{M}_{m, p}(\mathbb{C})\right)$.

At the geometric level, this action of the algebraic torus $\mathcal{H}$ comes from the left action of $\mathcal{H}$ on $\mathcal{M}_{m, p}(\mathbb{C})$ by Poisson isomorphisms via:

$$
\left(a_{1}, \ldots, a_{m}, b_{1}, \ldots, b_{p}\right) \cdot M:=\operatorname{diag}\left(a_{1}, \ldots, a_{m}\right)^{-1} \cdot M \cdot \operatorname{diag}\left(b_{1}, \ldots, b_{p}\right)^{-1} .
$$

This action of $\mathcal{H}$ on $\mathcal{M}_{m, p}(\mathbb{C})$ induces an action of $\mathcal{H}$ on the set $\operatorname{Sympl}\left(\mathcal{M}_{m, p}(\mathbb{C})\right)$ of symplectic leaves in $\mathcal{M}_{m, p}(\mathbb{C})$ (cf. [2, §0.1]). As in [2], we view the $\mathcal{H}$-orbit of a symplectic leaf $\mathcal{L}$ as the set-theoretic union $\bigcup_{h \in \mathcal{H}} h . \mathcal{L} \subseteq \mathcal{M}_{m, p}(\mathbb{C})$, rather than as the family $\{h . \mathcal{L} \mid h \in \mathcal{H}\}$. 
We denote the set of such orbits by $\mathcal{H}-\operatorname{Sympl}\left(\mathcal{M}_{m, p}(\mathbb{C})\right)$. These orbits were described by Brown, Yakimov and the first named author who obtained the following results.

We use the notation of [2] except that we replace $n$ by $p$. In particular, we set $N=m+p$. Let $w_{\circ}^{m}, w_{\circ}^{p}$ and $w_{\circ}^{N}$ denote the respective longest elements in $S_{m}, S_{p}$ and $S_{N}$, respectively, so that $w_{0}^{r}(i)=r+1-i$ for $i=1, \ldots, r$. Recall from equation (3.24) and Lemma 3.12 of [2] that

$$
w_{\circ}^{N} \mathcal{S}=S_{N}^{\geq\left(w_{\circ}^{p}, w_{\circ}^{m}\right)}:=\left\{w \in S_{N} \mid w \geq\left(w_{\circ}^{p}, w_{\circ}^{m}\right)\right\},
$$

where

$$
\left(w_{\circ}^{p}, w_{\circ}^{m}\right):=\left[\begin{array}{llllllll}
1 & 2 & \cdots & p & p+1 & p+2 & \cdots & p+m \\
p & p-1 & \cdots & 1 & p+m & p+m-1 & \cdots & p+1
\end{array}\right] .
$$

Theorem 2.1 [2, Theorems 3.9, 3.13, 4.2]

1. There are only finitely many $\mathcal{H}$-orbits of symplectic leaves in $\mathcal{M}_{m, p}(\mathbb{C})$, and they are smooth irreducible locally closed subvarieties.

2. The set $\mathcal{H}$-Sympl $\left(\mathcal{M}_{m, p}(\mathbb{C})\right)$ of orbits (partially ordered by inclusions of closures) is isomorphic to the set $S_{N}^{\geq\left(w_{0}^{p}, w_{\circ}^{m}\right)}$ with respect to the Bruhat order.

3. Each $\mathcal{H}$-orbit of symplectic leaves is defined by the vanishing and nonvanishing of certain sets of minors.

4. Each closure of an $\mathcal{H}$-orbit of symplectic leaves is defined by the vanishing of a certain set of minors.

For $y \in S_{N}^{\geq\left(w_{\circ}^{p}, w_{\circ}^{m}\right)}$, we denote by $\mathcal{P}_{y}$ the $\mathcal{H}$-orbit of symplectic leaves described in [2, Theorem 3.9].

These results have several consequences for the (potential) link between the Poisson structure of $\mathcal{O}\left(\mathcal{M}_{m, p}(\mathbb{C})\right)$ and the noncommutative structures of $\mathcal{O}_{q}\left(\mathcal{M}_{m, p}(\mathbb{C})\right)$ and $\mathcal{O}_{q}\left(\mathcal{M}_{m, p}(\mathbb{K})\right)$. For instance, combined with [18, Corollary 1.5], the theorem shows that the number of $\mathcal{H}$-orbits of symplectic leaves in $\mathcal{M}_{m, p}(\mathbb{C})$ is the same as the number of $\mathcal{H}$-prime ideals in $\mathcal{O}_{q}\left(\mathcal{M}_{m, p}(\mathbb{K})\right)$, and so the same as the number of $m \times p$ Cauchon diagrams.

In [9, Theorem 2.9], we extended the results of the previous theorem. More precisely, we defined, for each restricted permutation $w \in \mathcal{S}$, a family $\mathcal{M}(w)$ of minors and proved that a minor $[I \mid \Lambda]$ vanishes on $\overline{\mathcal{P}_{w_{o}^{N} w}}$ if and only if $[I \mid \Lambda] \in \mathcal{M}(w)$. (The conditions used to define $\mathcal{M}(w)$ will be given below in Definition 4.3, to define the corresponding family $\mathcal{M}_{q}(w)$ of quantum minors.)

To finish this section, let us mention that the symplectic leaves in $\mathcal{M}_{m, p}(\mathbb{C})$ are algebraic; that is, they are locally closed subvarieties of $\mathcal{M}_{m, p}(\mathbb{C})$. As a consequence, [8, Proposition 4.8] applies to our situation, so that there are only finitely many Poisson $\mathcal{H}$-primes in $\mathcal{O}\left(\mathcal{M}_{m, p}(\mathbb{C})\right)$. More precisely, the Poisson $\mathcal{H}$-primes in $\mathcal{O}\left(\mathcal{M}_{m, p}(\mathbb{C})\right)$ are in bijection with the $\mathcal{H}$-orbits of symplectic leaves in $\mathcal{M}_{m, p}(\mathbb{C})$, and the minors that belong to a Poisson $\mathcal{H}$-prime ideal are exactly those that vanish on the closure of the corresponding $\mathcal{H}$-orbit of symplectic leaves in $\mathcal{M}_{m, p}(\mathbb{C})$. Hence, the number of Poisson $\mathcal{H}$-primes in $\mathcal{O}\left(\mathcal{M}_{m, p}(\mathbb{C})\right)$ is the same as the number of $m \times p$ Cauchon diagrams, and the families of minors that belong to Poisson $\mathcal{H}$-primes are exactly the families $\mathcal{M}(w)$ with $w \in \mathcal{S}$.

In [9, Section 5], we constructed an (explicit) bijection between the set of $m \times p$ Cauchon diagrams and the set of Poisson $\mathcal{H}$-primes in $\mathcal{O}\left(\mathcal{M}_{m, p}(\mathbb{C})\right)$. As in [9, Theorem 5.3], we denote by $J_{C}^{\prime}$ the unique Poisson $\mathcal{H}$-prime associated to the Cauchon diagram $C$ under this bijection. 


\section{$3 q$-quantum matrices and the deleting-derivations algorithm}

For the remainder of this section, $K$ denotes a $\mathbb{K}$-algebra which is also a skew-field. Except where otherwise stated, all the matrices that are considered have their entries in $K$.

\section{$3.1 q$-quantum matrices}

Definition 3.1 (See [21, Chapter 4]) Let $M=\left(x_{i, \alpha}\right) \in \mathcal{M}_{m, p}(K)$. We say that $M$ is a $q$-quantum matrix if the following relations hold between the entries of $M$ :

$$
\begin{array}{lll}
x_{i, \beta} x_{i, \alpha}=q^{-1} x_{i, \alpha} x_{i, \beta}, & & (\alpha<\beta) ; \\
x_{j, \alpha} x_{i, \alpha}=q^{-1} x_{i, \alpha} x_{j, \alpha}, & & (i<j) ; \\
x_{j, \beta} x_{i, \alpha}=x_{i, \alpha} x_{j, \beta}, & & (i<j, \alpha>\beta) ; \\
x_{j, \beta} x_{i, \alpha}=x_{i, \alpha} x_{j, \beta}-\left(q-q^{-1}\right) x_{i, \beta} x_{j, \alpha}, & & (i<j, \alpha<\beta) .
\end{array}
$$

In order to define the deleting-derivations algorithm in the next section, we will need the following notation.

Notation 3.2 - We denote by $\leq$ the lexicographic ordering on $\mathbb{N}^{2}$. Recall that

$$
(i, \alpha) \leq(j, \beta) \Longleftrightarrow[(i<j) \text { or }(i=j \text { and } \alpha \leq \beta)] \text {. }
$$

- Set $E^{\circ}=(\llbracket 1, m \rrbracket \times \llbracket 1, p \rrbracket) \backslash\{(1,1)\}$ and $E=E^{\circ} \cup\{(m, p+1)\}$.

- Let $(j, \beta) \in E^{\circ}$. Then $(j, \beta)^{+}$denotes the smallest element (relative to $\leq$) of the set $\{(i, \alpha) \in E \mid(j, \beta)<(i, \alpha)\}$.

The deleting derivations/restoration algorithms will be applied to matrices that are not necessarily $q$-quantum matrices. However, the matrices involved do have reasonable commutation relations that lead to the following definition.

Definition 3.3 Let $M=\left(x_{i, \alpha}\right) \in \mathcal{M}_{m, p}(K)$ and let $(j, \beta) \in E$. We say that $M$ is a $(j, \beta)$ $q$-quantum matrix if the following relations hold between the entries of $M$. If $\left(\begin{array}{ll}x & y \\ z & t\end{array}\right)$ is any $2 \times 2$ sub-matrix of $M$, then

1. $y x=q^{-1} x y, \quad z x=q^{-1} x z, \quad z y=y z, \quad t y=q^{-1} y t, \quad t z=q^{-1} z t$.

2. If $t=x_{k, \gamma}$, then $t x= \begin{cases}x t & \text { (if }(k, \gamma) \geq(j, \beta)) \\ x t-\left(q-q^{-1}\right) y z & \text { (if }(k, \gamma)<(j, \beta)) .\end{cases}$

Note that our definitions of $q$-quantum matrix and $(j, \beta)$-q-quantum matrix differ slightly from those of [4, Definitions III.1.1 and III.1.3]. Because of this, we must interchange $q$ and $q^{-1}$ whenever carrying over results from [4], and we need a shift of index in the above definition. More precisely, Cauchon's definition of a $(j, \beta)$-q-quantum matrix [4, Definition III.1.3] matches, after switching $q$ and $q^{-1}$, our definition of a $(j, \beta)^{+}-q$-quantum matrix.

\subsection{The deleting-derivations algorithm}

Now, we recall the deleting-derivations algorithm (see [6], Convention 4.1.1 and [16], Conventions 2.2.3). This algorithm plays a central role in the study of the $\mathcal{H}$-prime ideals of the algebra of generic quantum matrices.

Convention 3.4 (Deleting-derivations algorithm) Let $M=\left(x_{i, \alpha}\right) \in \mathcal{M}_{m, p}(K)$ be a matrix. As $r$ runs over the set $E$, we define matrices $M^{(r)}:=\left(x_{i, \alpha}^{(r)}\right) \in \mathcal{M}_{m, p}(K)$ as follows. 
1. When $r=(m, p+1)$, the entries of the matrix $M^{(m, p+1)}$ are defined by $x_{i, \alpha}^{(m, p+1)}:=x_{i, \alpha}$ $\overline{\text { for all }(i, \alpha) \in \llbracket 1, m \rrbracket} \times \llbracket 1, p \rrbracket$.

2. Assume that $r=(j, \beta) \in E^{\circ}$ and that the matrix $M^{\left(r^{+}\right)}=\left(x_{i, \alpha}^{\left(r^{+}\right)}\right)$has already been defined. The entries $x_{i, \alpha}^{(r)}$ of the matrix $M^{(r)}$ are defined as follows.

(a) If $x_{j, \beta}^{\left(r^{+}\right)}=0$, then $x_{i, \alpha}^{(r)}=x_{i, \alpha}^{\left(r^{+}\right)}$for all $(i, \alpha) \in \llbracket 1, m \rrbracket \times \llbracket 1, p \rrbracket$.

(b) If $x_{j, \beta}^{\left(r^{+}\right)} \neq 0$ and $(i, \alpha) \in \llbracket 1, m \rrbracket \times \llbracket 1, p \rrbracket$, then

$$
x_{i, \alpha}^{(r)}= \begin{cases}x_{i, \alpha}^{\left(r^{+}\right)}-x_{i, \beta}^{\left(r^{+}\right)}\left(x_{j, \beta}^{\left(r^{+}\right)}\right)^{-1} x_{j, \alpha}^{\left(r^{+}\right)} & \text {if } i<j \text { and } \alpha<\beta \\ x_{i, \alpha}^{\left(r^{+}\right)} & \text {otherwise. }\end{cases}
$$

We say that $M^{(r)}$ is the matrix obtained from $M$ by applying the standard deleting-derivations algorithm at step $r$, and $x_{j, \beta}^{\left(r^{+}\right)}$is called the pivot at step $r$.

3. If $r=(1,2)$, then we set $t_{i, \alpha}:=x_{i, \alpha}^{(1,2)}$ for all $(i, \alpha) \in \llbracket 1, m \rrbracket \times \llbracket 1, p \rrbracket$. Observe that $x_{i, \alpha}^{(r)}=x_{i, \alpha}^{\left(r^{+}\right)}$for all $r \leq(i, \alpha)$, and so $t_{i, \alpha}=x_{i, \alpha}^{(i, \alpha)}=x_{i, \alpha}^{(i, \alpha)^{+}}$for all $(i, \alpha) \in E^{\circ}$.

When $m=p$, step 2 of the deleting-derivations algorithm can be written as $M^{(j, \beta)}=$ $\phi_{(m, j, \beta)}\left(M^{(j, \beta)^{+}}\right)$in the notation of [4, Sect. III.2.3].

Lemma 3.5 Let $(j, \beta) \in E$. If $M=\left(x_{i, \alpha}\right) \in \mathcal{M}_{m, p}(K)$ is a q-quantum matrix, then the matrix $M^{(j, \beta)}$ is $(j, \beta)$-q-quantum.

Proof When $m=p$, this follows from [4, Proposition III.2.3.1] by induction on $(j, \beta)$. The rectangular case is proved in the same manner. It also follows by applying the square case to a square matrix obtained from $M$ by adjoining suitable zero rows or columns.

\section{$3.3 \mathcal{H}$-invariant $q$-quantum matrices}

Before introducing the class of $q$-quantum matrices that will be of interest for us, let us give some notation for the quantum minors of a $q$-quantum matrix and the matrices obtained by applying the deleting-derivations algorithm.

Notation 3.6 Let $M=\left(x_{i, \alpha}\right) \in \mathcal{M}_{m, p}(K)$, and let $\delta=[I \mid \Lambda]_{q}(M)$ be a quantum minor of $M$. If $(j, \beta) \in E$, set

$$
\delta^{(j, \beta)}:=[I \mid \Lambda]_{q}\left(M^{(j, \beta)}\right)
$$

For $i \in I$ and $\alpha \in \Lambda$, set

$$
\delta_{\widehat{i}, \widehat{\alpha}}^{(j, \beta)}:=[I \backslash\{i\} \mid \Lambda \backslash\{\alpha\}]_{q}\left(M^{(j, \beta)}\right),
$$

while

$$
\delta_{\alpha \rightarrow \gamma}^{(j, \beta)}:=[I \mid \Lambda \cup\{\gamma\} \backslash\{\alpha\}]_{q}\left(M^{(j, \beta)}\right) \quad(\gamma \in \llbracket 1, p \rrbracket \backslash \Lambda)
$$

and

$$
\delta_{i \rightarrow k}^{(j, \beta)}:=[I \cup\{k\} \backslash\{i\} \mid \Lambda]_{q}\left(M^{(j, \beta)}\right) \quad(k \in \llbracket 1, m \rrbracket \backslash I) .
$$

In [16], the effect of the deleting-derivations algorithm on quantum minors was studied. Here we restrict our attention to a particular class of $q$-quantum matrices. 
Definition 3.7 A $q$-quantum matrix $M=\left(x_{i, \alpha}\right) \in \mathcal{M}_{m, p}(\mathbb{K})$ is said to be $\mathcal{H}$-invariant if, for any $(j, \beta) \in E^{\circ}$ and any quantum minor $\delta=\left[i_{1}, \ldots, i_{l} \mid \alpha_{1}, \ldots, \alpha_{l}\right]_{q}(M)$ of $M$ such that $\left(i_{l}, \alpha_{l}\right)<(j, \beta)$, we have:

$$
\delta^{(j, \beta)^{+}}=0 \Longrightarrow \delta^{(j, \beta)}=0 .
$$

This definition is motivated by the fact proved in [15, Proposition 3.1.4.5] that, if $J$ is an $\mathcal{H}$-prime ideal of the algebra $\mathcal{O}_{q}\left(\mathcal{M}_{m, p}(\mathbb{K})\right)$, then the matrix $\left(X_{i, \alpha}+J\right)$ (whose entries are the canonical images of the generators of $\mathcal{O}_{q}\left(\mathcal{M}_{m, p}(\mathbb{K})\right)$ modulo the $\mathcal{H}$-prime $\left.J\right)$ is an $\mathcal{H}$-invariant $q$-quantum matrix. For the convenience of the reader, another proof of this result is presented in Corollary 3.9.

Also, in the case where $\mathbb{K}=\mathbb{C}$, it was proved in [9, Theorem 5.4] that, if $J^{\prime}$ is a Poisson $\mathcal{H}$-prime ideal of $\mathcal{O}\left(\mathcal{M}_{m, p}(\mathbb{C})\right)$, then the matrix $\left(Y_{i, \alpha}+J^{\prime}\right)$ over $\mathcal{O}\left(\mathcal{M}_{m, p}(\mathbb{C})\right) / J^{\prime}$ is an $\mathcal{H}$-invariant 1-quantum matrix.

\subsection{Effect of the deleting-derivations algorithm on quantum minors}

The aim of this section is to obtain a characterisation of the quantum minors of $M^{(j, \beta)^{+}}$that are equal to zero in terms of the quantum minors of $M^{(j, \beta)}$ that are equal to zero, for an $\mathcal{H}$-invariant $q$-quantum matrix $M$. The first step does not require $\mathcal{H}$-invariance.

Proposition 3.8 Let $M=\left(x_{i, \alpha}\right) \in \mathcal{M}_{m, p}(K)$ be a q-quantum matrix and $r=(j, \beta) \in E^{\circ}$. Set $u:=x_{j, \beta}^{(j, \beta)^{+}}$and let $\delta=\left[i_{1}, \ldots, i_{l} \mid \alpha_{1}, \ldots, \alpha_{l}\right]_{q}(M)$ be a quantum minor of $M$ with $\left(i_{l}, \alpha_{l}\right)<(j, \beta)$. Assume that $u \neq 0, i_{l}<j$ and $\alpha_{h}<\beta<\alpha_{h+1}$ for some $h \in \llbracket 1, l \rrbracket$. (By convention, $\alpha_{l+1}=p+1$.) Then

$$
\delta^{(j, \beta)^{+}} u=\delta^{(j, \beta)} u+q \delta_{\alpha_{h} \rightarrow \beta}^{\left(j, \alpha_{h}\right)} x_{j, \alpha_{h}}^{\left(j, \alpha_{h}\right)} .
$$

Proof We proceed by induction on $l+1-h$. If $l+1-h=1$, then $h=l$ and $\alpha_{l}<\beta$. It follows from [16, Proposition 2.2.8] that

$$
\delta^{(j, \beta)^{+}} u=\delta^{(j, \beta)} u-\sum_{k=1}^{l}(-q)^{(l+1)-k} \delta_{i_{k} \rightarrow j}^{(j, \beta)} x_{i_{k}, \beta}^{(j, \beta)} .
$$

Moreover it follows from [16, Proposition 2.2.8] that

$$
\delta_{i_{k} \rightarrow j}^{(j, \beta)}=\delta_{i_{k} \rightarrow j}^{(j, \beta-1)}=\cdots=\delta_{i_{k} \rightarrow j}^{\left(j, \alpha_{l}\right)^{+}} .
$$

Then we deduce from [6, Propositions 4.1.1 and 4.1.2] that

$$
\delta_{i_{k} \rightarrow j}^{(j, \beta)}=\delta_{\widehat{i_{k}}, \widehat{\alpha_{l}}}^{\left(j, \alpha_{l}\right)} x_{j, \alpha_{l}}^{\left(j, \alpha_{l}\right)} .
$$

As $x_{i_{k}, \beta}^{(j, \beta)}=x_{i_{k}, \beta}^{(j, \beta-1)}=\cdots=x_{i_{k}, \beta}^{\left(j, \alpha_{l}\right)}$ by construction, we obtain

$$
\delta^{(j, \beta)^{+}} u=\delta^{(j, \beta)} u-\sum_{k=1}^{l}(-q)^{(l+1)-k} \delta_{\widehat{i_{k}}, \widehat{\alpha_{l}}}^{\left(j, \alpha_{l}\right)} x_{j, \alpha_{l}}^{\left(j, \alpha_{l}\right)} x_{i_{k}, \beta}^{\left(j, \alpha_{l}\right)} .
$$

As $i_{k}<j, \alpha_{l}<\beta$ and the matrix $M^{\left(j, \alpha_{l}\right)}$ is $\left(j, \alpha_{l}\right)$-q-quantum, $x_{j, \alpha_{l}}^{\left(j, \alpha_{l}\right)}$ and $x_{i_{k}, \beta}^{\left(j, \alpha_{l}\right)}$ commute, so that

$$
\delta^{(j, \beta)^{+}} u=\delta^{(j, \beta)} u-\sum_{k=1}^{l}(-q)^{(l+1)-k} \delta_{\widehat{i_{k}}, \widehat{\alpha_{l}}}^{\left(j, \alpha_{l}\right)} x_{i_{k}, \beta}^{\left(j, \alpha_{l}\right)} x_{j, \alpha_{l}}^{\left(j, \alpha_{l}\right)}
$$


Hence, by using a $q$-Laplace expansion [10, Corollary A.5, Equation (A.6)], we obtain

$$
\delta^{(j, \beta)^{+}} u=\delta^{(j, \beta)} u+q \delta_{\alpha_{l} \rightarrow \beta}^{\left(j, \alpha_{l}\right)} x_{j, \alpha_{l}}^{\left(j, \alpha_{l}\right)},
$$

as desired.

Now let $l+1-h>1$, and assume the result holds for smaller values of $l+1-h$. Note that $\beta<\alpha_{h+1} \leq \alpha_{l}$. Expand the quantum minor $\delta^{(j, \beta)^{+}}$along its last column [10, Corollary A.5, Equation (A.5)], to get

$$
\delta^{(j, \beta)^{+}}=\sum_{k=1}^{l}(-q)^{k-l} x_{i_{k}, \alpha_{l}}^{(j, \beta)^{+}} \delta_{\widehat{i_{k}}, \widehat{\alpha_{l}}}^{(j, \beta)^{+}} .
$$

The value corresponding to $l+1-h$ for the minors $\delta_{\widehat{i_{k}}, \widehat{\alpha_{l}}}^{(j, \beta)^{+}}$is $l-h$, and so the induction hypothesis applies. We obtain

$$
\delta_{\overparen{i_{k}}, \widehat{\alpha_{l}}}^{(j, \beta)^{+}} u=\delta_{\widehat{i_{k}}, \widehat{\alpha_{l}}}^{(j, \beta)} u+q \delta_{\substack{\hat{i}_{k}, \widehat{\alpha_{l}} \\ \alpha_{h} \rightarrow \beta}}^{\left(j, \alpha_{h}\right)} x_{j, \alpha_{h}}^{\left(j, \alpha_{h}\right)}
$$

for $k \in \llbracket 1, l \rrbracket$. As $x_{i_{k}, \alpha_{l}}^{(j, \beta)^{+}}=x_{i_{k}, \alpha_{l}}^{(j, \beta)}=\cdots=x_{i_{k}, \alpha_{l}}^{\left(j, \alpha_{h}\right)}$ by construction, we obtain

$$
\begin{aligned}
\delta^{(j, \beta)^{+}} u & =\sum_{k=1}^{l}(-q)^{k-l} x_{i_{k}, \alpha_{l}}^{(j, \beta)} \delta_{\hat{i}_{k}, \widehat{\alpha_{l}}}^{(j, \beta)} u+q \sum_{k=1}^{l}(-q)^{k-l} x_{i_{k}, \alpha_{l}}^{\left(j, \alpha_{h}\right)} \delta_{\substack{i_{k}, \widehat{\alpha_{l}} \\
\alpha_{h} \rightarrow \beta}}^{\left(j, \alpha_{h}\right)} x_{j, \alpha_{h}}^{\left(j, \alpha_{h}\right)} \\
& =\delta^{(j, \beta)} u+q \delta_{\alpha_{h} \rightarrow \beta}^{\left(j, \alpha_{h}\right)} x_{j, \alpha_{h}}^{\left(j, \alpha_{h}\right)}
\end{aligned}
$$

by two final $q$-Laplace expansions [10, Corollary A.5, Equation (A.5)]. This concludes the induction step.

Corollary 3.9 Let $J$ be an $\mathcal{H}$-prime ideal of the algebra $R=\mathcal{O}_{q}\left(\mathcal{M}_{m, p}(\mathbb{K})\right)$. Then the matrix $\left(X_{i, \alpha}+J\right) \in \mathcal{M}_{m, p}(R / J)$ is an $\mathcal{H}$-invariant q-quantum matrix.

Proof Set $x_{i, \alpha}=X_{i, \alpha}+J$ for all $(i, \alpha)$ and $M=\left(x_{i, \alpha}\right) \in \mathcal{M}_{m, p}\left(F_{J}\right)$, where $F_{J}$ denotes the skew-field of fractions of the noetherian domain $R / J$. Clearly, $M$ is a $q$-quantum matrix. Let $(j, \beta) \in E^{\circ}$ and let $\delta=\left[i_{1}, \ldots, i_{l} \mid \alpha_{1}, \ldots, \alpha_{l}\right]_{q}(M)$ be a quantum minor of $M$ such that $\left(i_{l}, \alpha_{l}\right)<(j, \beta)$. Assume that $\delta^{(j, \beta)^{+}}=0$. We need to prove that $\delta^{(j, \beta)}=0$. If $\delta^{(j, \beta)}=\delta^{(j, \beta)^{+}}$, then there is nothing to do; so assume that $\delta^{(j, \beta)} \neq \delta^{(j, \beta)^{+}}$. In this case, it follows from [16, Proposition 2.2.8] that $u=t_{j, \beta}=x_{j, \beta}^{(j, \beta)^{+}} \neq 0$ and $i_{l}<j$ while $\alpha_{h}<\beta<\alpha_{h+1}$ for some $h \in \llbracket 1, l \rrbracket$. Thus, Proposition 3.8 implies that

$$
\delta^{(j, \beta)^{+}} u=\delta^{(j, \beta)} u+q \delta_{\alpha_{h} \rightarrow \beta}^{\left(j, \alpha_{h}\right)} x_{j, \alpha_{h}}^{\left(j, \alpha_{h}\right)} .
$$

Hence,

$$
0=\delta^{(j, \beta)} u+q \delta_{\alpha_{h} \rightarrow \beta}^{\left(j, \alpha_{h}\right)} t_{j, \alpha_{h}}
$$

In order to conclude, observe that each $x_{i, \alpha}^{(j, \beta)}=t_{i, \alpha}+Q_{i, \alpha}$ where the element $Q_{i, \alpha}$ lies in the algebra

$$
\left.L:=\mathbb{K}\left\langle t_{k, \gamma}^{ \pm 1}\right|(k, \gamma)<(j, \beta) \text { and } t_{k, \gamma} \neq 0\right\rangle,
$$

and that each $x_{i, \alpha}^{\left(j, \alpha_{h}\right)}=t_{i, \alpha}+Q_{i, \alpha}^{\prime}$ where

$$
\left.Q_{i, \alpha}^{\prime} \in \mathbb{K}\left\langle t_{k, \gamma}^{ \pm 1}\right|(k, \gamma)<\left(j, \alpha_{h}\right) \text { and } t_{k, \gamma} \neq 0\right\rangle \subseteq L
$$


(This can be proved by an easy induction, similar to [16, Lemme 3.5.4].) Hence, $\delta^{(j, \beta)}$ and $\delta_{\alpha_{h} \rightarrow \beta}^{\left(j, \alpha_{h}\right)} t_{j, \alpha_{h}}$ both belong to $L$. Finally, we note that [17, Theorem 3.7], which was proved for the case $\mathbb{K}=\mathbb{C}$, holds for the general coefficient field $\mathbb{K}$ (with the same proof). This result implies that the powers of $u=t_{j, \beta}$ are linearly independent over $L$. It follows that $\delta^{(j, \beta)}=0$, as desired.

We now restrict our attention to the case where $M$ is an $\mathcal{H}$-invariant $q$-quantum matrix. In this case, we deduce from [16, Proposition 2.2.8] and Proposition 3.8 the following characterisation of the quantum minors of $M^{(j, \beta)^{+}}$that are equal to zero in terms of the quantum minors of $M^{(j, \beta)}$ that are equal to zero.

Proposition 3.10 Let $M=\left(x_{i, \alpha}\right) \in \mathcal{M}_{m, p}(K)$ be an $\mathcal{H}$-invariant q-quantum matrix and $(j, \beta) \in E^{\circ}$. Set $u:=x_{j, \beta}^{(j, \beta)^{+}}$. Let $\delta=\left[i_{1}, \ldots, i_{l} \mid \alpha_{1}, \ldots, \alpha_{l}\right]_{q}(M)$ be a quantum minor of $M$ with $\left(i_{l}, \alpha_{l}\right)<(j, \beta)$.

1. Assume that $u=0$. Then $\delta^{(j, \beta)^{+}}=0$ if and only if $\delta^{(j, \beta)}=0$.

2. Assume that $u \neq 0$. If $i_{l}=j$, or if $\beta \in\left\{\alpha_{1}, \ldots, \alpha_{l}\right\}$, or if $\beta<\alpha_{1}$, then $\delta^{(j, \beta)^{+}}=0$ if and only if $\delta^{(j, \beta)}=0$.

3. Assume that $u \neq 0, i_{l}<j$ and $\alpha_{h}<\beta<\alpha_{h+1}$ for some $h \in \llbracket 1, l \rrbracket$. Then $\delta^{(j, \beta)^{+}}=0$ if and only if $\delta^{(j, \beta)}=0$ and either $\delta_{\alpha_{h} \rightarrow \beta}^{\left(j, \alpha_{h}\right)}=0$ or $x_{j, \alpha_{h}}^{\left(j, \alpha_{h}\right)}=0$.

Proof (1) and (2) follow from [16, Proposition 2.2.8]. (3) follows from the previous proposition and the fact that $M$ is $\mathcal{H}$-invariant.

\section{Poisson $\mathcal{H}$-primes of $\mathcal{O}\left(\mathcal{M}_{m, p}(\mathbb{C})\right)$ versus $\mathcal{H}$-primes in $\mathcal{O}_{q}\left(\mathcal{M}_{m, p}(\mathbb{K})\right)$}

We are now in position to study the quantum minors that belong to a given $\mathcal{H}$-prime in the algebra $R=\mathcal{O}_{q}\left(\mathcal{M}_{m, p}(\mathbb{K})\right)$.

Associated to each $m \times p$ Cauchon diagram $C$ is an $\mathcal{H}$-prime $J_{C}$ of $R$ determined as follows [6]. Via the deleting-derivations algorithm, $R$ is connected to a quantum affine space $\bar{R}$ with generators $T_{i, \alpha}$ for $(i, \alpha) \in \llbracket 1, m \rrbracket \times \llbracket 1, p \rrbracket$, and there is a canonical embedding $\varphi: \operatorname{Spec}(R) \rightarrow \operatorname{Spec}(\bar{R})$. The set $\left\{T_{i, \alpha} \mid(i, \alpha) \in C\right\}$ generates an $\mathcal{H}$-prime ideal of $\bar{R}$, and $J_{C}$ is the inverse image of this $\mathcal{H}$-prime under $\varphi$. Moreover, the rule $C \mapsto J_{C}$ gives a bijection from $\mathcal{C}_{m, p}$ onto $\mathcal{H}-\operatorname{Spec}(R)$. (See [17, Section 2] for a summary of the details.) It is the reverse connection - going from $J_{C}$ back to $C$ - that is important for the proof of the next theorem. To describe it, set $x_{i, \alpha}:=X_{i, \alpha}+J_{C}$ for $(i, \alpha) \in \llbracket 1, m \rrbracket \times \llbracket 1, p \rrbracket$, consider the $q$-quantum matrix $M_{C}:=\left(x_{i, \alpha}\right) \in \mathcal{M}_{m, p}\left(R / J_{C}\right)$, and apply the deleting-derivations algorithm to $M_{C}$. For all $(i, \alpha)$, (the $\mathbb{K}$-version of) [17, Theorem 3.7] shows that

$$
x_{i, \alpha}^{(1,2)}=0 \Longleftrightarrow(i, \alpha) \in C .
$$

Consequently, $x_{i, \alpha}^{(i, \alpha)}=0 \Longleftrightarrow(i, \alpha) \in C$ (recall that $\left.x_{i, \alpha}^{(1,2)}=x_{i, \alpha}^{(i, \alpha)}\right)$.

Associated to $C$ is also a Poisson $\mathcal{H}$-prime $J_{C}^{\prime}$ of $\mathcal{O}\left(\mathcal{M}_{m, p}(\mathbb{C})\right)$ which can be described in an analogous fashion. However, it is given in [9, Section 5] using the (Poisson) restoration algorithm (the inverse of the deleting-derivations algorithm), in the following way. First, let $A_{C}$ denote the polynomial ring

$$
\mathbb{C}\left[t_{i, \alpha} \mid(i, \alpha) \in(\llbracket 1, m \rrbracket \times \llbracket 1, p \rrbracket) \backslash C\right],
$$

and set $t_{i, \alpha}=0 \in A_{C}$ for $(i, \alpha) \in C$. A specific Poisson bracket is defined on $A_{C}$, and the restoration algorithm is applied to the matrix $\left(t_{i, \alpha}\right) \in \mathcal{M}_{m, p}\left(A_{C}\right)$. This leads to a Poisson algebra 
$A_{C}^{\prime}:=A_{C}^{(m, p+1)}$ with generators $y_{i, \alpha}$, such that there is a Poisson algebra homomorphism $\varphi_{C}: \mathcal{O}\left(\mathcal{M}_{m, p}(\mathbb{C})\right) \rightarrow A_{C}^{\prime}$ sending $Y_{i, \alpha} \mapsto y_{i, \alpha}$ for all $(i, \alpha)$. The Poisson $\mathcal{H}$-prime ideal $J_{C}^{\prime}$ associated to $C$ is defined as $J_{C}^{\prime}:=\operatorname{ker}\left(\varphi_{C}\right)$, and we identify $A_{C}^{\prime}$ with $\mathcal{O}\left(\mathcal{M}_{m, p}(\mathbb{C})\right) / J_{C}^{\prime}$. The rule $C \mapsto J_{C}^{\prime}$ defines a bijection from $\mathcal{C}_{m, p}$ onto $\mathcal{H}$-PSpec $\left(\mathcal{O}\left(\mathcal{M}_{m, p}(\mathbb{C})\right)\right)$.

Application of the (Poisson) deleting-derivations algorithm [9, Convention B.2] to the matrix $M_{C}^{\prime}:=\left(y_{i, \alpha}\right) \in \mathcal{M}_{m, p}\left(A_{C}^{\prime}\right)$ leads back to the matrix $\left(t_{i, \alpha}\right)$, so that $t_{i, \alpha}=y_{i, \alpha}^{(1,2)}$ for all $(i, \alpha) \in \llbracket 1, m \rrbracket \times \llbracket 1, p \rrbracket$. In view of the definition of the $t_{i, \alpha}$, we thus have

$$
y_{i, \alpha}^{(1,2)}=0 \Longleftrightarrow(i, \alpha) \in C .
$$

Consequently, just as with (3), $y_{i, \alpha}^{(i, \alpha)}=0 \Longleftrightarrow(i, \alpha) \in C$.

Theorem 4.1 Let $C$ be an $m \times p$ Cauchon diagram. Let $J_{C}$ be the corresponding $\mathcal{H}$-prime in $\mathcal{O}_{q}\left(\mathcal{M}_{m, p}(\mathbb{K})\right)$, and $J_{C}^{\prime}$ the corresponding Poisson $\mathcal{H}$-prime in $\mathcal{O}\left(\mathcal{M}_{m, p}(\mathbb{C})\right)$.

Then, a quantum minor $[I \mid \Lambda]_{q}$ belongs to $J_{C}$ if and only if the corresponding minor $[I \mid \Lambda]$ belongs to $J_{C}^{\prime}$.

Proof Define the matrix $M_{C}:=\left(x_{i, \alpha}\right)$ as above, and observe that a quantum minor $\delta$ belongs to $J_{C}$ if and only if it corresponds to a quantum minor of $M_{C}$ that is equal to zero. Moreover, it follows from Corollary 3.9 that $M_{C}$ is an $\mathcal{H}$-invariant $q$-quantum matrix. In particular, Proposition 3.10 applies to $M_{C}$.

Similarly, define the matrix $M_{C}^{\prime}:=\left(y_{i, \alpha}\right)$ as above, and observe that a minor $\delta$ belongs to $J_{C}^{\prime}$ if and only if it corresponds to a minor of $M_{C}^{\prime}$ that is equal to zero. Moreover, [9, Proposition 3.15] applies to $M_{C}^{\prime}$.

So it is sufficient to prove that a quantum minor of $M_{C}$ is equal to zero if and only if the corresponding minor of $M_{C}^{\prime}$ is equal to zero.

We prove, by induction on $(j, \beta) \in E$, that a quantum minor $\delta^{(j, \beta)}:=[I \mid \Lambda]_{q}\left(x_{i, \alpha}^{(j, \beta)}\right)$ of $M_{C}^{(j, \beta)}$, where $I=\left\{i_{1}, \ldots, i_{l}\right\}$ and $\Lambda=\left\{\alpha_{1}, \ldots, \alpha_{l}\right\}$ with $\left(i_{l}, \alpha_{l}\right)<(j, \beta)$, is equal to zero if and only if the corresponding minor $\Delta^{(j, \beta)}:=[I \mid \Lambda]\left(y_{i, \alpha}^{(j, \beta)}\right)$ of $M_{C}^{\prime}{ }^{(j, \beta)}$ is equal to zero.

The case $(j, \beta)=(1,2)$ follows from the fact that we start with the same Cauchon diagram. Then the induction step follows easily from the fact that one can apply Proposition 3.10 to $M_{C}$ and [9, Proposition 3.15] to $M_{C}^{\prime}$. Note that the induction step mimics the proof of [9, Theorem 3.16]. However, for the convenience of the reader we provide details.

We prove that $\delta^{(j, \beta)}=0$ implies that $\Delta^{(j, \beta)}=0$. The reverse implication is proved similarly.

Assume first that $(j, \beta)=(1,2)$. In this case, we have to prove that if $x_{1,1}^{(1,2)}=0$, then $y_{1,1}^{(1,2)}=0$. Assume that $x_{1,1}^{(1,2)}=0$. Then $(1,1) \in C$ by (3), whence it follows from (4) that $y_{1,1}^{(1,2)}=0$, as desired.

Now let $(j, \beta) \in E^{\circ}$, and assume the result proved at step $(j, \beta)$. Suppose that $\delta^{(j, \beta)^{+}}=0$. In order to prove that $\Delta^{(j, \beta)^{+}}=0$, we consider several cases. Keep in mind that $t_{j, \beta}=$ $x_{j, \beta}^{(j, \beta)}=x_{j, \beta}^{(j, \beta)^{+}}$.

- Assume that $\left(i_{l}, \alpha_{l}\right)=(j, \beta)$. We distinguish between two cases.

$\bullet$ If $t_{j, \beta}=x_{j, \beta}^{(j, \beta)}=0$, then $(j, \beta) \in C$ by (3). Hence, it follows from (4) that $y_{j, \beta}^{(j, \beta)}=0$, and so we deduce from [9, Proposition 3.10] that $\Delta^{(j, \beta)^{+}}=0$, as required. 
• If $t_{j, \beta}=x_{j, \beta}^{(j, \beta)} \neq 0$, then it follows from [6, Proposition 4.1.2] that $0=\delta^{(j, \beta)^{+}}=$ $\delta_{\widehat{j}, \widehat{\beta}}^{(j, \beta)} x_{j, \beta}^{(j, \beta)}$, so that $\delta_{\hat{j}, \widehat{\beta}}^{(j, \beta)}=0$. Hence, it follows from the induction hypothesis that $\Delta_{\hat{j}, \widehat{\beta}}^{(j, \beta)}=0$. As $\Delta^{(j, \beta)^{+}}=\Delta \widehat{j}_{\hat{j}, \widehat{\beta}}^{(j, \beta)} y_{j, \beta}^{(j, \beta)}$, by [9, Proposition 3.10], it follows that $\Delta^{(j, \beta)^{+}}=0$, as required.

- Assume that $\left(i_{l}, \alpha_{l}\right)<(j, \beta)$. We distinguish between three cases (corresponding to the three cases of Proposition 3.10).

• Assume that $t_{j, \beta}=0$. As we are assuming that $\delta^{(j, \beta)^{+}}=0$, it follows from Proposition 3.10 that $\delta^{(j, \beta)}=\delta^{(j, \beta)^{+}}=0$. Hence, we deduce from the induction hypothesis that $\Delta^{(j, \beta)}=0$. On the other hand, as $t_{j, \beta}=0$, we have $(j, \beta) \in C$ and so $y_{j, \beta}^{(j, \beta)}=0$. Thus, it follows from [9, Proposition 3.15] that $\Delta^{(j, \beta)^{+}}=\Delta^{(j, \beta)}=0$, as desired.

$\bullet \quad$ Assume that $t_{j, \beta} \neq 0$, and that $i_{l}=j$, or that $\beta \in\left\{\alpha_{1}, \ldots, \alpha_{l}\right\}$, or that $\beta<\alpha_{1}$. As we are assuming that $\delta^{(j, \beta)^{+}}=0$, it follows from Proposition 3.10 that $\delta^{(j, \beta)}=$ $\delta^{(j, \beta)^{+}}=0$. Hence, we deduce from the induction hypothesis that $\Delta^{(j, \beta)}=0$. On the other hand, as $t_{j, \beta} \neq 0$, we have $(j, \beta) \notin C$ and so $y_{j, \beta}^{(j, \beta)} \neq 0$. Moreover, as $i_{l}=j$, or $\beta \in\left\{\alpha_{1}, \ldots, \alpha_{l}\right\}$, or $\beta<\alpha_{1}$, it follows from [9, Proposition 3.15] that $\Delta^{(j, \beta)^{+}}=\Delta^{(j, \beta)}=0$, as desired.

•- Assume that $t_{j, \beta} \neq 0$ and $i_{l}<j$, while $\alpha_{h}<\beta<\alpha_{h+1}$ for some $h \in \llbracket 1, l \rrbracket$. Then, as in the previous case, we have $y_{j, \beta}^{(j, \beta)} \neq 0$. Moreover, it follows from Proposition 3.10 that $\delta^{(j, \beta)^{+}}=0$ implies $\delta^{(j, \beta)}=0$ and either $\delta_{\alpha_{h} \rightarrow \beta}^{\left(j, \alpha_{h}\right)}=0$ or $x_{j, \alpha_{h}}^{\left(j, \alpha_{h}\right)}=0$. Hence, we deduce from the induction hypothesis that $\Delta^{(j, \beta)}=0$ and either $\Delta_{\alpha_{h} \rightarrow \beta}^{\left(j, \alpha_{h}\right)}=0$ or $y_{j, \alpha_{h}}^{\left(j, \alpha_{h}\right)}=0$. Finally, it follows from [9, Proposition 3.15] that $\Delta^{(j, \beta)^{+}}=0$, as desired.

Theorem 4.2 Let $\mathcal{F}$ be a family of minors in the coordinate ring $\mathcal{O}\left(\mathcal{M}_{m, p}(\mathbb{C})\right)$, and let $\mathcal{F}_{q}$ be the corresponding family of quantum minors in $\mathcal{O}_{q}\left(\mathcal{M}_{m, p}(\mathbb{K})\right)$. Then the following are equivalent:

1. The totally nonnegative cell associated to $\mathcal{F}$ in $\mathcal{M}_{\bar{m}}^{\geq 0}, p(\mathbb{R})$ is non-empty.

2. $\mathcal{F}$ is the set of all minors that vanish on the closure of some $\mathcal{H}$-orbit of symplectic leaves in $\mathcal{M}_{m, p}(\mathbb{C})$.

3. $\mathcal{F}_{q}$ is the set of all quantum minors that belong to some $\mathcal{H}$-prime in $\mathcal{O}_{q}\left(\mathcal{M}_{m, p}(\mathbb{K})\right)$.

Proof The equivalence of (1) and (2) is proved in [9, Theorem 6.2].

On the other hand, as discussed in Sect. 2.2 above, the Poisson $\mathcal{H}$-primes in $\mathcal{O}\left(\mathcal{M}_{m, p}(\mathbb{C})\right)$ are in bijection with the $\mathcal{H}$-orbits of symplectic leaves in $\mathcal{M}_{m, p}(\mathbb{C})$, and the minors that belong to a Poisson $\mathcal{H}$-prime ideal are exactly those that vanish on the closure of the corresponding $\mathcal{H}$-orbit of symplectic leaves in $\mathcal{M}_{m, p}(\mathbb{C})$. Thus, (2) holds if and only if $\mathcal{F}$ is the set of all minors that belong to some Poisson $\mathcal{H}$-prime ideal in $\mathcal{O}\left(\mathcal{M}_{m, p}(\mathbb{C})\right)$. The remaining equivalence, $(2) \Longleftrightarrow(3)$, now follows from Theorem 4.1 .

The families of minors that vanish on the closure of an $\mathcal{H}$-orbit of symplectic leaves have been explicitly described in [9, Theorem 2.11]. They are parametrised by the set $\mathcal{S}$ of restricted permutations. Theorem 4.2 shows that the quantum analogues of these families provide the families of quantum minors that belong to $\mathcal{H}$-primes in $\mathcal{O}_{q}\left(\mathcal{M}_{m, p}(\mathbb{K})\right)$.

Let us now be more precise. In [9, Definition 2.6] a family of minors $\mathcal{M}(w)$ is defined for each $w \in \mathcal{S}$. The corresponding definition for families of quantum minors is given below. 
Definition 4.3 For $w \in \mathcal{S}$, define $\mathcal{M}_{q}(w)$ to be the set of those quantum minors $[I \mid \Lambda]_{q}$ of $\mathcal{O}_{q}\left(\mathcal{M}_{m, p}(\mathbb{K})\right)$, with $I \subseteq \llbracket 1, m \rrbracket$ and $\Lambda \subseteq \llbracket 1, p \rrbracket$, that satisfy at least one of the following conditions.

1. $I\left\lfloor\leq w_{\circ}^{m} w(L)\right.$ for all $L \subseteq \llbracket 1, p \rrbracket \cap w^{-1} \llbracket 1, m \rrbracket$ such that $|L|=|I|$ and $L \leq \Lambda$.

2. $m+\Lambda\left\lfloor z w w_{\circ}^{N}(L)\right.$ for all $L \subseteq \llbracket 1, m \rrbracket \cap w_{\circ}^{N} w^{-1} \llbracket m+1, N \rrbracket$ such that $|L|=|\Lambda|$ and $L \leq I$.

3. There exist $1 \leq r \leq s \leq p$ such that $|\Lambda \cap \llbracket r, s \rrbracket|>\left|\llbracket r, s \rrbracket \backslash w^{-1} \llbracket m+r, m+s \rrbracket\right|$.

4. There exist $1 \leq r \leq s \leq m$ such that $|I \cap \llbracket r, s \rrbracket|>\left|w_{\circ}^{N} \llbracket r, s \rrbracket \backslash w^{-1} w_{\circ}^{m} \llbracket r, s \rrbracket\right|$.

We are now in position to establish the following result which answers positively a conjecture of Brown, Yakimov and the first named author in the case where char $(\mathbb{K})=0$ and $q$ is transcendental over $\mathbb{Q}$.

Theorem 4.4 1. Let $J$ be an $\mathcal{H}$-prime ideal of $\mathcal{O}_{q}\left(\mathcal{M}_{m, p}(\mathbb{K})\right)$. Then there exists $w \in \mathcal{S}$ such that $\mathcal{M}_{q}(w)$ is exactly the set of those quantum minors that belong to $J$.

2. Assume that $\operatorname{char}(\mathbb{K})=0$ and $q$ is transcendental over $\mathbb{Q}$. Then we have:

$$
\mathcal{H}-\operatorname{Spec}\left(\mathcal{O}_{q}\left(\mathcal{M}_{m, p}(\mathbb{K})\right)\right)=\left\{\left\langle\mathcal{M}_{q}(w)\right\rangle \mid w \in \mathcal{S}\right\} .
$$

Proof (1) is a consequence of Theorem 4.2 and [9, Theorem 2.11]. Then (2) follows from (1) and Theorem 1.5.

A different approach to the second part of this result, applicable to many quantized coordinate algebras, has been recently and independently developed by Yakimov in [23] (see [23, Theorem 5.5]).

Acknowledgements The results in this paper were announced during the mini-workshop "Non-negativity is a quantum phenomenon" that took place at the Mathematisches Forschungsinstitut Oberwolfach, 1-7 March 2009, [20]; we thank the director and staff of the MFO for providing the ideal environment for this stimulating meeting. We also thank Konni Rietsch, Laurent Rigal, Lauren Williams and Milen Yakimov for discussions and comments concerning this paper both at the workshop and at other times. Finally, we thank Karel Casteels for sending us his preprint [3].

Open Access This article is distributed under the terms of the Creative Commons Attribution Noncommercial License which permits any noncommercial use, distribution, and reproduction in any medium, provided the original author(s) and source are credited.

\section{References}

1. Brown, K.A., Goodearl, K.R.: Lectures on Algebraic Quantum Groups, Advanced Courses in Mathematics. CRM Barcelona, Birkhäuser Verlag, Basel (2002)

2. Brown, K.A., Goodearl, K.R., Yakimov, M.: Poisson structures on affine spaces and flag varieties.I. Matrix affine Poisson space. Adv. Math. 206(2), 567-629 (2006)

3. Casteels, K.: A graph theoretic method for determining generating sets of prime ideals in $\mathcal{O}_{q}\left(\mathcal{M}_{m, n}(\mathbb{C})\right)$. arXiv:0907.1617

4. Cauchon, G.: Quotients premiers de $O_{q}\left(\mathcal{M}_{n}(k)\right)$. J. Algebra 180(2), 530-545 (1996)

5. Cauchon, G.: Effacement des dérivations et spectres premiers des algèbres quantiques. J. Algebra 260(2), 476-518 (2003)

6. Cauchon, G.: Spectre premier de $O_{q}\left(M_{n}(k)\right)$ : image canonique et séparation normale. J. Algebra 260(2), 519-569 (2003)

7. Fulton, W.: Flags, Schubert polynomials, degeneracy loci, and determinantal formulas. Duke Math. J. 65(3), 381-420 (1992)

8. Goodearl, K.R.: A Dixmier-Moeglin equivalence for Poisson algebras with torus actions. In: Algebra and its Applications, pp. 131-154. Contemp. Math., vol. 419, Amer. Math. Soc., Providence (2006) 
9. Goodearl, K.R., Launois, S., Lenagan, T.H.: Totally nonnegative cells and matrix Poisson varieties. arXiv:0905.3631

10. Goodearl, K.R., Lenagan, T.H.: Prime ideals invariant under winding automorphisms in quantum matrices. Int. J. Math. 13(5), 497-532 (2002)

11. Goodearl, K.R., Lenagan, T.H.: Winding-invariant prime ideals in quantum $3 \times 3$ matrices. J. Algebra 260(2), 657-687 (2003)

12. Goodearl, K.R., Letzter, E.S.: Prime factor algebras of the coordinate ring of quantum matrices. Proc. Am. Math. Soc. 121(4), 1017-1025 (1994)

13. Goodearl, K.R., Letzter, E.S.: The Dixmier-Moeglin equivalence in quantum coordinate rings and quantized Weyl algebras. Trans. Am. Math. Soc. 352(3), 1381-1403 (2000)

14. Goodearl, K.R., Yakimov, M.: Poisson structures on affine spaces and flag varieties. II. Trans. Am. Math. Soc 361, 5753-5780 (2009)

15. Launois, S.: Idéaux premiers $\mathcal{H}$-invariants de l'algèbre des matrices quantiques. Thèse de Doctorat, Université de Reims (2003)

16. Launois, S.: Les idéaux premiers invariants de $O_{q}\left(\mathcal{M}_{m, p}(\mathbb{C})\right)$. J. Algebra 272(1), 191-246 (2004)

17. Launois, S.: Generators for $\mathcal{H}$-invariant prime ideals in $O_{q}\left(\mathcal{M}_{m, p}(\mathbb{C})\right)$, Proc. Edinb. Math. Soc. (2) 47 no. 1, 163-190 (2004)

18. Launois, S.: Combinatorics of $\mathcal{H}$-primes in quantum matrices. J. Algebra 309(1), 139-167 (2007)

19. Launois, S., Lenagan, T.H., Rigal, L.: Prime ideals in the quantum Grassmannian. Selecta Math. (N.S.) 13(4), 697-725 (2008)

20. Mini-Workshop: Non-negativity is a quantum phenomenon. Abstracts from the mini-workshop held 1-7 March 2009. Organised by S. Launois and T.H. Lenagan. Oberwolfach Reports 6, no. 14 (2009)

21. Parshall, B., Wang, J.P.: Quantum linear groups. Mem. Am. Math. Soc. 89, 439, vi+157 (1991)

22. Postnikov, A.: Total positivity, grassmannians, and networks. math.CO/0609764

23. Yakimov, M.: Invariant prime ideals in quantizations of nilpotent Lie algebras. Proc. London Math. Soc. doi:10.1112/plms/pdq006 